\title{
Desenvolvimento e Avaliação de uma Metodologia de Aprendizagem Ativa Apoiada pelo uso de QR Code para Ensino de Banco de Dados
}

\author{
Ronney Moreira de Castro, Sean Wolfgand Matsui Siqueira \\ Programa de Pós-Graduação em Informática (PPGI) \\ Universidade Federal do Estado do Rio de Janeiro (UNIRIO) \\ Av. Pasteur, 456 - Urca - Rio de Janeiro - RJ - Brasil \\ ronney.castro@uniriotec.br, sean@uniriotec.br
}

\begin{abstract}
Considering the development of information and communication technologies and the reality of the students, the traditional lectures tend to be monotonous and uninteresting. Active Learning is an option to this type of class, bringing techniques closer to the students, resulting in greater motivation and involvement on their part. This paper presents a technique based on QR Code as a support to teaching. The authors applied the technique in a Database course of Information Systems. The goal is to provide students with a more practical and engaging activity encompassing knowledge explored in the course and also help teachers in choosing a different methodology to be adopted in their classes.
\end{abstract}

Resumo. Diante do desenvolvimento das tecnologias da Informação $e$ comunicação e a realidade dos alunos, a aula tradicional expositiva tende a ser monótona e desinteressante. A Aprendizagem Ativa (AA) é uma opção a este tipo de aula, trazendo técnicas mais próximas dos alunos, resultando em uma maior motivação e envolvimento por parte deles. Este artigo apresenta uma técnica baseada em QR Code como apoio ao ensino. Os autores aplicaram a técnica em uma disciplina de Banco de Dados do curso de Sistemas de Informação. $O$ objetivo é levar aos alunos uma atividade mais prática e envolvente englobando conhecimentos vistos na disciplina e também auxiliar professores na escolha de uma metodologia diferente a ser adotada em suas aulas.

\section{Introdução}

A sala de aula tradicional, que apoia a sociedade industrial, baseia-se na figura central do docente, que é o detentor e disseminador do conhecimento e os alunos, por sua vez, têm pouco ou nenhum espaço para suas opiniões [Araújo et al. 2015]. O tipo de aula mais utilizado é a tradicional ou mais conhecida como expositiva. Nela, o professor é aquele que possui o conhecimento e passa o mesmo aos alunos, que devem dominar informações no lugar de realmente aprenderem a ter suas próprias opiniões [Thomas e Brown 2011].

Os currículos da sala de aula tradicional fundamentam-se, principalmente, no material elaborado que irá definir o que e como ensinar, acomodando as práticas escolares e estabelecendo uma fronteira pedagógica que distancia professores de alunos, restringindo a forma de pensar [Carvalho; Nevado; Menezes 2005]. Observa-se, assim, que os métodos de ensino adotados mostram-se ineficazes para a formação dos alunos e, 
um dos maiores desafios da área da Educação está relacionado a quais práticas devem ser implantadas em sala de aula que possam favorecer os processos de aprendizagem [Acosta; Reategui; Behar 2016].

Na sociedade contemporânea, o pensamento crítico, a colaboração, a capacidade de solucionar problemas e a tomada de decisões são habilidades fundamentais [Acosta; Reategui; Behar 2016]. A aprendizagem está diretamente associada a um processo que ocorre em etapas de construção e reconstrução e é fundamental que os indivíduos possam interagir com outros sujeitos [Piaget 1977]. O aprendizado, então, necessita ter relação com o concreto, ou seja, o aluno deve vivenciar situações reais apoiado por um mediador/coordenador (professor) e construir seu conhecimento através dessas experiências [Behar 2011].

A Aprendizagem Ativa (AA) é uma alternativa em relação às aulas tradicionais podendo complementar as mesmas e motivar os discentes para um estudo mais detalhado, além de proporcionar uma maior retenção do conhecimento [Bonwell e Eison 1991] [Meyers e Jones 1993]. Segundo Acharya et al. (2015) várias áreas fazem uso da AA para o ensino de conteúdos e a Computação recentemente também tem feito uso de tal técnica [Mitchell; Petter; Harris 2017].

Este artigo apresenta uma forma de usar o QR Code como técnica de AA na disciplina de Banco de Dados (BD). O objetivo é levar os alunos a uma busca por informações contidas nos códigos e, juntar as mesmas para resolver uma atividade proposta. Os códigos contêm informações importantes tais como tabelas, atributos, relacionamentos, códigos SQL entre outros. Com isso, os alunos poderão fixar conceitos importantes aprendidos na disciplina de BD.

A técnica foi utilizada em duas turmas do $4^{\circ}$ período de um Curso de Bacharelado em Sistemas de Informação, nos anos 2016 e 2017 e avaliada através da aplicação de um questionário e também da percepção dos autores durante a aplicação no laboratório. A dinâmica da técnica e os resultados da avaliação são apresentados no texto.

O restante do artigo está organizado em mais cinco seções. A seção 2 apresenta o conceito de Aprendizagem Ativa e sua utilização em cursos na área de Computação. A seção 3 descreve a técnica de QR Code propriamente dita, sua preparação, assim como a forma utilizada para aplicação em sala de aula. Os resultados alcançados estão na seção 4 e, por fim, a conclusão do artigo na seção 5 .

\section{Aprendizagem Ativa}

A Aprendizagem Ativa (AA) surgiu em 1990 e provou ser uma excelente alternativa para auxiliar os alunos em um maior envolvimento com o aprendizado [Bonwell e Eison 1991] [Meyers e Jones 1993]. Diversas áreas têm feito uso da AA tais como, por exemplo, Biologia, Computação, Direito, Medicina, entre outras [Acharya et al. 2015].

Allen e Tanner (2005) descrevem a AA como a aquisição de novos conteúdos de forma a construir seu significado e com a oportunidade de reiterá-lo a outros, além disso, enfatiza a interação entre os discentes e o docente. No lugar de simplesmente ficarem ouvindo as aulas expositivas, os alunos têm inúmeras oportunidades de participar de atividades e, principalmente, receber o feedback imediato sobre elas [Gleason et al. 2011]. 
Prince (2004) define a AA como uma "atividade de sala de aula que exige que os alunos façam algo diferente de ouvir e tomar notas". Morgan et al. (2005) relatam que não há uma definição clara na literatura para AA, mas existem algumas comumente utilizadas: i) "Experiência de aprendizagem multidirecional na qual a aprendizagem ocorre de professor para aluno, aluno para professor e aluno para aluno"; ii) "Qualquer coisa que envolve os alunos em fazer coisas e pensar sobre as coisas que estão fazendo"; iii) "É definido em contraste com o pior do ensino tradicional, no qual os professores ativamente apresentam a informação e os alunos passivamente a recebem"; iv) "Qualquer método instrucional que envolva os alunos no processo de aprendizagem"; v) "Qualquer estilo de ensino que maximize a participação dos alunos no processo de aprendizagem". Mitchell; Petter; Harris (2017), afirmam que AA "são exercícios introduzidos na sala de aula para incentivar o pensamento e a participação dos alunos em um esforço para envolvê-los no processo de aprendizagem".

Para que uma atividade possa ser classificada como "ativa" é necessário que o aluno esteja ativamente envolvido na mesma e aplique o conhecimento construído de forma a fazer sentido para sua aprendizagem [Linnenbrink e Pintrich 2004]. As técnicas de AA incrementam as aulas e motivam os discentes, além de proporcionar uma maior assimilação do conhecimento. Alunos motivados são mais propensos a participar das aulas e, portanto, isso auxilia a sustentar o interesse e o prazer dos discentes pela aprendizagem [Barkley 2010].

Atividades como animações, dinâmicas de grupo, estudos de caso, jogos, quizzes, simuladores, vídeos são exemplos de AA. Segundo Thongmak (2017), a aprendizagem no século XXI tem-se baseado muito na AA. Para o autor, a AA é uma mudança na forma de aprendizagem permitindo aos docentes basear suas aulas em tarefas ou perguntas de forma a auxiliar os discentes a compreender melhor os conceitos envolvidos. Pesquisas recentes apoiam que os alunos valorizam a AA e a interação entre pares e que, estratégias como salas de aula invertidas, aprendizagem baseada em equipe, aprendizagem baseada em problemas e aprendizagem baseada em casos, podem ter excelentes resultados entre os alunos [Thongmak 2017].

Como qualquer estratégia, a AA não é a solução de todos os problemas de ensino-aprendizagem. Suas técnicas podem ser aplicadas levando em consideração os resultados de forma a tornar o processo de aprendizagem mais expressivo para o aluno [Drake 2012].

\subsection{Aprendizagem Ativa em Sistemas de Informação/Computação}

Mitchell; Petter; Harris (2017) elaboraram uma revisão de artigos publicados relacionados ao uso de AA em Sistemas de Informação. Os autores conduziram uma revisão sistemática da literatura usando a string de busca "active learning" $\mathrm{E}$ "information systems", no período de janeiro de 2000 a julho de 2016 em revistas acadêmicas da ABI/INFORM Collection, Informing Sciences Institute library e a eLibrary da Association for Information Systems (AIS). Foram selecionados 49 artigos e identificadas vinte tipos diferentes de técnicas agrupadas em 5 categorias: (1) apresentações visuais; (2) projetos colaborativos de estudantes; (3) interação tecnológica; (4) avaliação; e (5) jogos.

Acharya et al. (2015) utilizaram ferramentas de AA tais como exercícios em classe, estudos de caso e vídeo desenvolvidos em parceria com empresas de software 
com o objetivo de compreender os tópicos relacionados à Engenharia de Software (validação e verificação de software, engenharia de requisitos, revisões, inspeções, gerenciamento de configuração e testes). Massey; Brown; Johnston (2005) fazem uso de jogos como técnica de AA para incentivar os alunos a revisar os conteúdos, além de envolvê-los melhor nas aulas. São propostos um jogo web de perguntas e respostas sobre diversos assuntos aplicado em sala de aula, e palavras cruzadas como tarefas para casa. Ramiller (2002) apresenta o Virtual Interactive Project (VIP), que faz um intercâmbio entre projetos de campo e projetos baseados em texto. Nele os alunos atuam em equipes como se fossem empresas de desenvolvimento e o professor como um "cliente virtual", disposto a responder aos questionamentos para o desenvolvimento de uma aplicação.

Dong et al. (2017) relata que é muito difícil para os docentes saber se todos os alunos estão envolvidos ou não na aula. Os discentes recebem informações do professor, porém ocorre pouca ou nenhuma interação, o que os leva a enfrentar dificuldade em manter a concentração. Uma forma de envolver o aluno e professor é através do uso de uma tecnologia projetada para isso. $\mathrm{O}$ trabalho apresenta uma ferramenta que faz uso de "Sistemas de Resposta Instantânea" (Instant Response Systems - IRSs) ou "clickers" que possibilita aos alunos solicitar uma pausa na aula quando necessário. Além disso, possibilita ao docente saber se o material de aprendizagem utilizado está sendo passado de forma rápida ou com dificuldade para os alunos compreenderem.

Castro e Siqueira (2017) apresentam algumas técnicas de AA utilizadas em um curso de Bacharelado em Sistemas de Informação. As técnicas foram: i) Uso de Quizzes, uma ferramenta desenvolvida pelos próprios alunos contendo um tabuleiro virtual que fica projetado usando um equipamento de Datashow em sala de aula. Os alunos devem se dividir em equipes e responder as perguntas sobre o assunto Engenharia de Software. Existem casas "coringas" que correspondem a prendas (ex: "imitar o professor X"; "cantar uma canção", "imitar o bicho Y" etc.) que servem para dar um caráter mais descontraído ao jogo; ii) Boneco de Jornal, utilizado para ensino do conceito de processos [Castro e Souza 2016]. Os alunos têm que se dividir em equipes e construir um boneco utilizando folhas de jornal e tesoura. Porém, existem fases a serem cumpridas. Em uma primeira fase, os grupos não têm comunicação com os outros, o que leva o boneco ficar como um "monstro", levando os alunos a entender, analogamente, que é importante a comunicação dentro de uma empresa de desenvolvimento de software, por exemplo. São aplicadas mais outras fases nas quais o boneco vai melhorando aos poucos, mostrando também que o processo não é estático, mas sim deve estar em melhoria contínua; iii) Jogo Agility Scrum para ensino do Scrum [Castro et al. 2017] no qual os alunos também se dividem em equipes e determinam os papéis dentro das mesmas: Scrum Master ou desenvolvedor. O professor faz o papel de Product Owner. As equipes têm que montar um circuito eletrônico que possui como componentes leds, baterias, suporte de bateria, resistores, fios, botões de acionamento e protoboard. Os Sprints são fixados no quadro da sala adaptado a um quadro de Kanbam e são sequenciais, ou seja, a equipe deve terminar o primeiro sprint, entregar o mesmo, logo depois construir o sprint seguinte. Os sprints são uma sequência de funcionalidades do circuito ficando mais complexo na medida que forem implementados. Ganha o jogo o grupo que terminar todo o circuito e entregar o mesmo em pleno funcionamento. A técnica também faz uso práticas do Movimento Maker, mais especificamente de circuitos eletrônicos. Este movimento tem como conceito fundamental, aprender com a 
prática. No trabalho também foi citado o uso de QR Codes, porém de forma sucinta. Aqui detalhamos a técnica com o objetivo de divulgar a mesma para docentes que queiram adotá-la em suas aulas.

\section{Uso de QR Code na Disciplina de Banco de Dados}

A disciplina Banco de Dados está presente em praticamente todos os cursos na área de Computação. Muitas vezes divide-se a mesma em duas partes: uma que envolve somente a modelagem em si, usada para descrição de dados no nível conceitual (Modelo Conceitual) e outra sobre a implementação propriamente dita (Modelo Físico), que envolve o aprendizado da linguagem SQL para que o Banco de Dados seja criado em um Gerenciador de Banco de Dados (SGBD).

A técnica aqui proposta envolve o conhecimento tanto do modelo conceitual quanto do modelo físico, permitindo ao professor trabalhar com os alunos uma atividade para fixar a matéria lecionada. Para isso é utilizado QR Code, uma evolução dos códigos de barras no qual as informações são ordenadas em uma matriz de duas dimensões, conforme figura 1. Os QR Codes são utilizados largamente nos dias de hoje. Algumas de suas aplicações mais conhecidas são revistas, campanhas publicitárias, aplicativos, bilhetes de passagem etc.

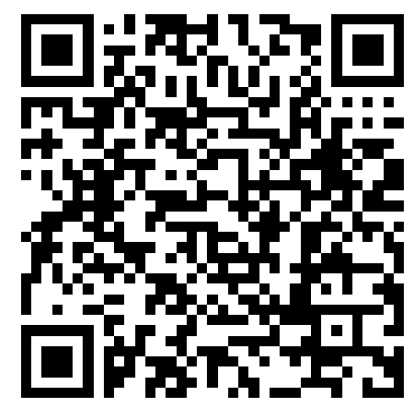

Figura 1. Exemplo de um QR Code [Elaborado pelos autores]

Em uma busca na literatura foram encontradas algumas aplicações usando QR Code para fins acadêmicos. Porém, não foi encontrado nenhum trabalho relacionado ao uso de QR Code com Banco de Dados. Os detalhes da técnica elaborada usando os códigos, assim como sua preparação são mostrados na Tabela 1.

Tabela 1. Detalhes e preparação para aplicação da técnica

\begin{tabular}{|c|l|}
\hline Objetivo & $\begin{array}{l}\text { Mostrar de forma divertida conceitos aprendidos na disciplina Banco de Dados: } \\
\text { Diagrama entidade-relacionamento (DER), Diagrama de tabelas relacionais (DTR), } \\
\text { relacionamento entre tabelas, cardinalidade, tabelas, atributos, tipo de atributos } \\
\text { (integer, char, varchar etc.). }\end{array}$ \\
\hline Participantes & Todos os alunos da sala de forma individual. \\
\hline Tempo & De 90 a 100 minutos. Em geral duas aulas germinadas. \\
\hline Local & $\begin{array}{l}\text { Usar um Laboratório que contenha PCs para a turma toda. Pode-se usar também dois } \\
\text { alunos por PC. Além disso, é necessário ter instalado nas máquinas um SGBD (SQL } \\
\text { Server, MYSQL, por exemplo) e um editor de textos. }\end{array}$ \\
\hline Material & $\begin{array}{l}\text { 20 folhas de papel A4 que deverão conter os QR Codes da tarefa. Celulares que } \\
\text { possuam câmera (Smartphones). Um aplicativo de leitura de QR Codes, } \\
\text { preferencialmente gratuito. Por conveniência, para o professor, é importante também } \\
\text { deixar impresso o que significa cada QR Code. Fita adesiva para fixar as folhas nas } \\
\text { paredes ou outro local. O material para aplicação da técnica pode ser solicitado por } \\
\text { e-mail aos autores ou acessado nesse link: <https://goo.gl/LgnbtH> }\end{array}$ \\
\hline
\end{tabular}



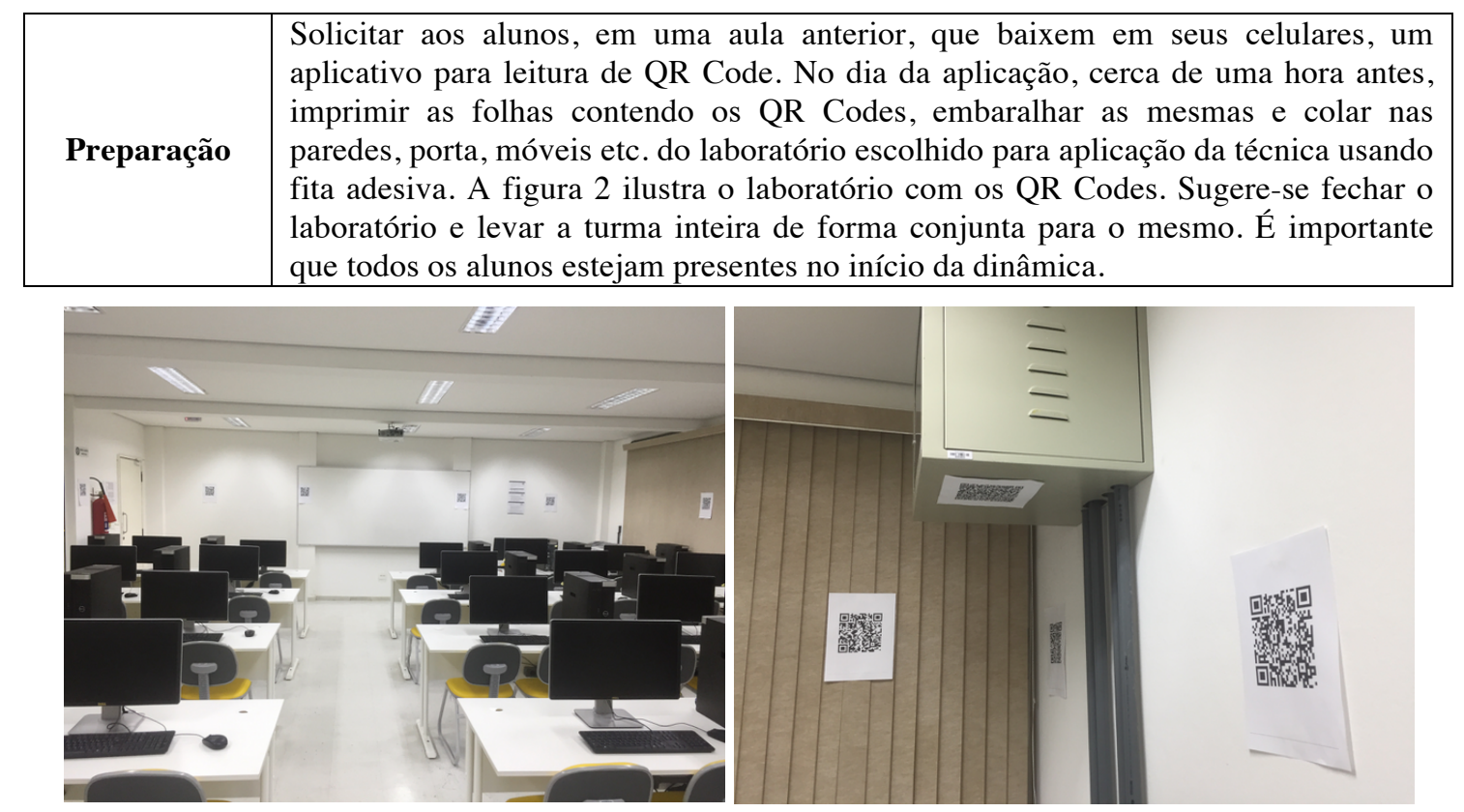

Figura 2. Distribuição das folhas com QR Code no laboratório

\subsection{Descrição}

Ao chegar no laboratório os alunos verão os códigos espalhados, o que já os deixará curiosos sobre o que farão naquela aula, além de gerar uma curiosidade sobre o que significa cada um dos códigos das folhas. O professor deve então explicar que existe um exercício a ser feito e que o texto e script do mesmo deverão ser entregues até o final da aula. Para isso será necessário fazer a leitura aleatória dos QR Codes localizados nas folhas. Logo após é dado o início por parte do professor e todos podem fazer a leitura dos códigos.

Existem folhas com trechos do enunciado do exercício (Exemplo: "Uma firma vende produtos de limpeza, e deseja melhor controlar os produtos que vende, seus clientes e os pedidos."), informações de cardinalidades (Exemplo: "Guarda-se igualmente a informação dos pedidos feitos pelos clientes. Cada pedido possui um número e guarda-se a data de elaboração do pedido. Cada pedido pode envolver de um a vários produtos, e para cada produto, indica-se a quantidade deste pedida."), partes de tabelas (Exemplo: "CodCliente char(3) Not Null, Nome varchar(50), Endereco varchar(50)"), tabela inteiras (Exemplo: "CodCliente char(3) Not Null, Nome varchar(50), Endereco varchar(50), Telefone varchar(15), Status char(5), LimiteCredito float $(10,2)$ "), folhas coringa, que introduzem obstáculos a serem ser tratados pelos alunos (Exemplo: "Esse QR Code não tem nenhuma informação. Vá para outro QR Code"), dicas (Exemplo: "É necessário juntar as partes de texto para saber o enunciado da tarefa").

O objetivo final não é ter um vencedor, mas que todos montem o exercício com seu enunciado e também façam o script de criação do Banco de Dados.

\subsection{Aplicação}

A técnica foi aplicada em duas turmas do $4^{\circ}$ período de um curso de Bacharelado em Sistemas de Informação da cidade de Juiz de Fora - MG nos anos de 2016 e 2017. Na 
instituição de ensino superior escolhida, a disciplina de Banco de Dados é dividida em duas partes: uma referente à Modelagem de Banco de Dados, no $3^{\circ}$ período e outra referente a Implementação de Banco de Dados no $4^{\circ}$ período. Todos os alunos já haviam cursado a parte de modelagem conceitual de Banco de Dados e já sabiam como criar as tabelas usando um script SQL. A Figura 3 mostra os participantes em 2016 e a Figura 4 os de 2017.
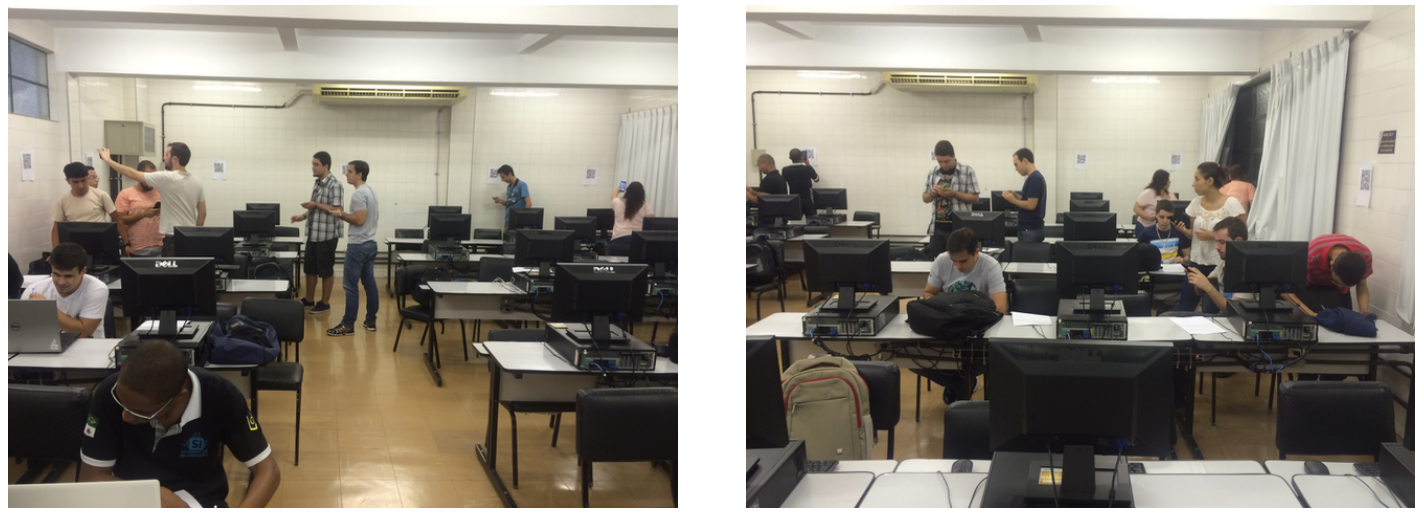

Figura 3. Alunos da turma de 2016 fazendo leitura dos QR Codes no laboratório
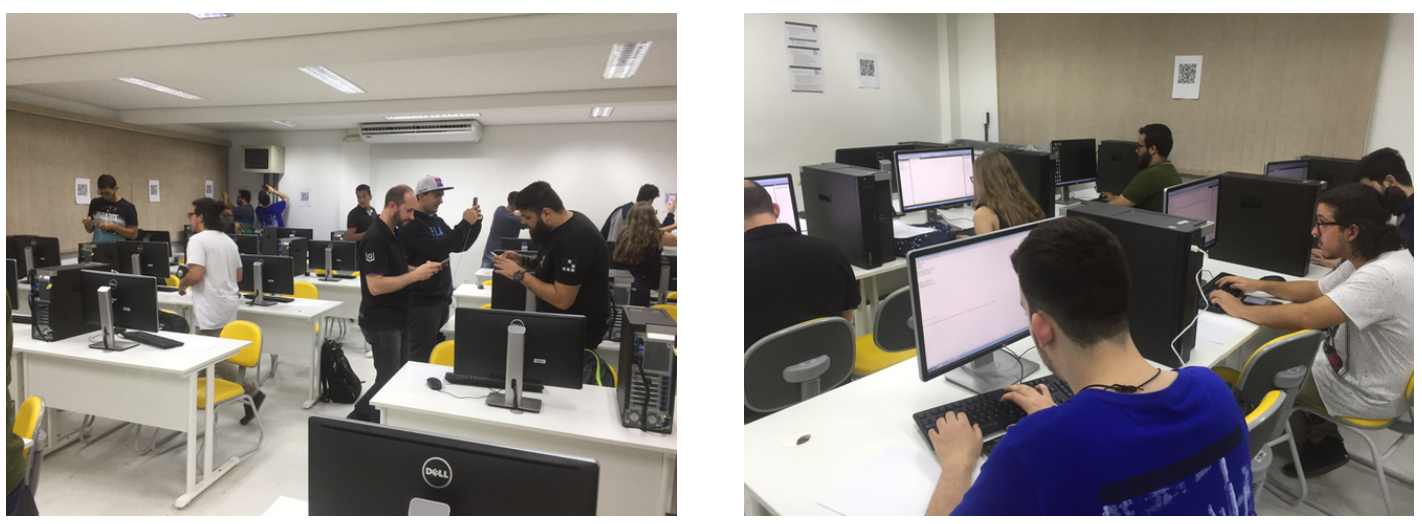

Figura 4. Alunos da turma de 2017 fazendo leitura dos QR Codes no laboratório

\section{Resultados}

Foi possível perceber através da observação dos participantes durante a tarefa, uma grande motivação principalmente por eles estarem utilizando seus celulares durante a atividade, para a coleta das informações necessárias e não para uso em redes sociais, por exemplo. Percebeu-se também um grande envolvimento durante todo o tempo de aplicação da atividade. Isso mostra que uma técnica de AA proporciona dinamismo, curiosidade envolvimento e satisfação ao final de sua aplicação.

O questionário utilizado no trabalho de Castro e Souza [2016], com uma pequena modificação na questão 1 , foi aplicado com o objetivo de medir o nível de aceitação da técnica. O mesmo foi composto de 25 questões que obedeciam a escala: 1 (ruim), 2 (razoável), 3 (bom), 4 (muito bom) e 5 (excelente). Na turma de 2016, 8 alunos responderam o questionário e na turma de 2017, 7 alunos. Por conta de limitação de espaço, questões do questionário foram omitidas assim como as respostas. Todas as questões e respostas podem ser acessadas no endereço: <https://goo.gl/LgnbtH>

Algumas questões foram destacadas. 
i) "Estou satisfeito com a atividade apresentada porque foi uma prática diferente das aulas convencionais" mostrou que tanto os alunos de 2016 quanto os de 2017 ficaram satisfeitos com a técnica e concordam que é uma atividade diferente das atividades tradicionais usadas em sala de aula. A figura 5 ilustra os resultados, sendo à esquerda os alunos de 2016 e à direita os alunos de 2017.
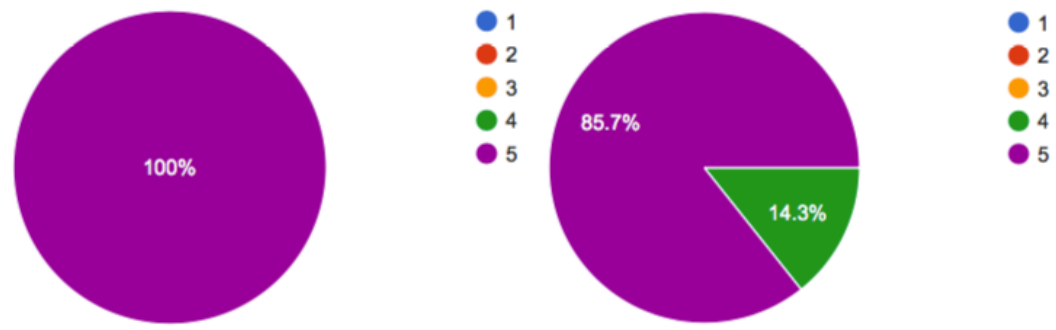

Figura 5. Respostas dos alunos da turma de 2016 e 2017, respectivamente, quanto a satisfação com a atividade diferente das usadas em aulas tradicionais

ii) "A utilização da atividade me fez ficar atento à aula ministrada". A figura 6, esquerda mostra os alunos de 2016 com cerca de $87,5 \%$ de aceitação e, à direita os alunos de 2017 com 100\% de aceitação. Percebe-se que foi detectado um outlier na turma de 2016 referente a 1(um) aluno que marcou a opção 1. Em análise posterior se observou tratar de um caso de questões particulares e não rejeição específica à técnica. Pode-se concluir que a técnica elaborada auxilia os alunos a ficarem mais atentos durante a aula.
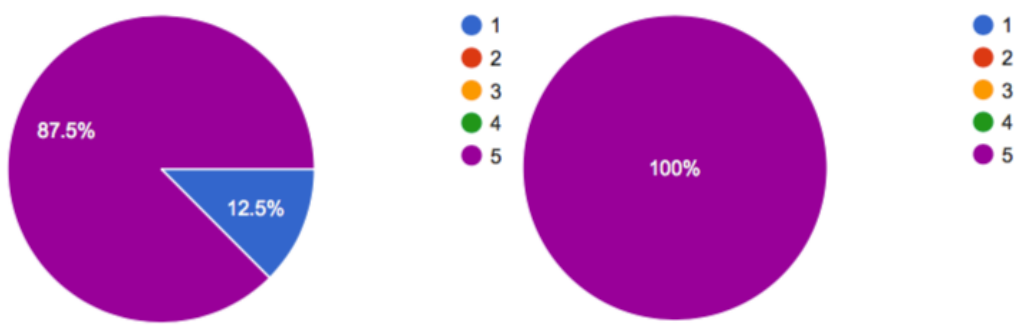

Figura 6. Respostas dos alunos da turma de 2016 e 2017 respectivamente quanto a atenção à aula ministrada

iii) "A atividade contribuiu para a minha aprendizagem na disciplina" mostra que os alunos também concordam que a atividade auxiliou sua aprendizagem na disciplina, ou seja, o uso de uma técnica AA pode contribuir para o aprendizado. A figura 7 ilustra os resultados obtidos (a direita os alunos de 2016 e a esquerda os alunos de 2017). Aqui também pode ser visualizado novamente o outlier na turma de 2016.
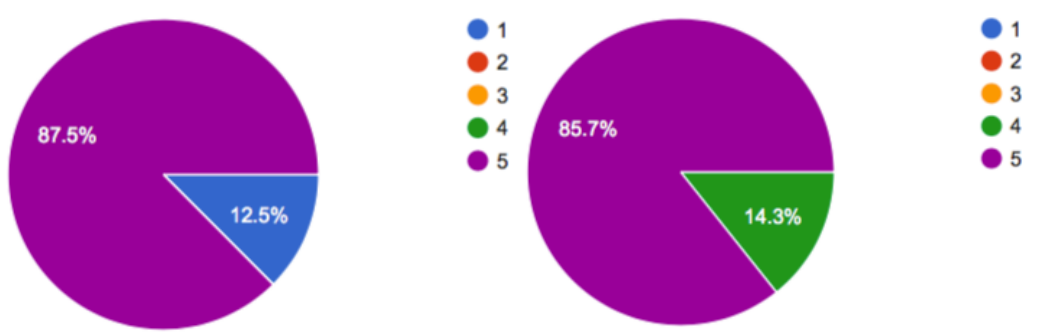

Figura 7. Respostas dos alunos da turma de 2016 e 2017 respectivamente quanto a contribuição da técnica para sua aprendizagem 


\section{Conclusão}

As aulas tradicionais (expositivas) tendem a não prender a atenção dos alunos. $\mathrm{O}$ uso da Aprendizagem Ativa (AA) pode mudar este cenário permitindo um melhor aprendizado do conteúdo lecionado nas aulas.

Este trabalho apresentou uma técnica de AA que pode ser utilizada por docentes da disciplina Banco de Dados (BD), de forma a completar o conteúdo abordado em sala de aula. Essa técnica mostrou-se eficaz em uma experiência com alunos de $4^{\circ}$ período de um curso de Bacharelado em Sistemas de Informação nos anos 2016 e 2017. A técnica não é limitada somente ao uso na disciplina de $\mathrm{BD}$, podendo também ser aplicada em outras disciplinas como, por exemplo, Análise Orientada a Objetos.

Deste modo, conclui-se que uma atividade didático-pedagógica diferenciada, ou mais especificamente uma atividade de AA, pode apoiar ensino-aprendizagem dos alunos, além de mantê-los motivados durante as aulas.

\section{Referências}

Acharya, S., Manohar, P. A., Wu, P., Schilling, W., Ansari, A. (2015). Integrated Active Learning Tools for Enhanced Pedagogy in a Software Engineering Course. Computers in Education Journal, 7(2), 17-28.

Acosta, O., Reategui, E., Behar, P. A. (2016). Recomendação de Conteúdo em um Ambiente Colaborativo de Aprendizagem Baseada em Projetos. Congresso Brasileiro de Informática e Educação - CBIE. Uberlândia.

Allen, D., Tanner, K. (2005). Infusing active learning into the large-enrollment Biology class: seven strategies, from the simple to complex. Cell Biol. Educ. 4(4), 262-268

Araújo, R. A., Santos, R.A.A., Farias, R. S., Franca, R. S., Silva, T. S. C., Vasconcelos, R. L., Tedesco, P., Padilha, M. A., Belian, R. B. (2015) Investigação sobre Inovações Pedagógicas Protagonizadas por Docentes em uma Instituição e Ensino Universitário no Brasil. In: CINDU2015 - IV Congresso Internacional de Docência Universitária. Vigo. Anais do CINDU 2015.

Barkley, E.: Student Engagement Techniques. Jossey-Bass, San Francisco (2010)

Behar, P. A. (2011). Constructing Pedagogical Models for E-Learning. International Journal of Advanced Corporate Learning, 4(3), 16-22.

Bonwell, C. C., Eison, J. A. (1991). Active learning: Creating excitement in the classroom. Association for the Study of Higher Education, Washington, DC.

Carvalho, M. J. S., Nevado, R. A., Menezes, C. S.. (2005). Arquiteturas Pedagógicas para Educação a Distância: Concepções e Suporte Telemático. SBIE - Simpósio Brasileiro de Informática na Educação, 351-360.

Castro, R. M., Souza, G. S. (2016). O Uso de Recursos Lúdicos para o Ensino de Processos em Engenharia de Software. 24 ${ }^{\circ}$ WEI - Workshop sobre Educação em Computação, XXXVI Congresso da Sociedade Brasileira de Computação, Porto Alegre. 
Castro, R. M. et al. (2016). AGILITY SCRUM - Um Jogo para Ensino da Metodologia SCRUM. $25^{\circ}$ WEI - Workshop sobre Educação em Computação, XXXVI Congresso da Sociedade Brasileira de Computação, São Paulo.

Castro, R. M., Siqueira, S. W. M. (2017). Aprendizagem Ativa em Sistemas de Informação: Novas Técnicas Propostas e Reflexões sobre as Experiências. $13^{\circ}$ SBSI Simpósio Brasileiro de Sistemas de Informação, Lavras - MG.

Dong, J. J., Hwang, W. Y., Shadiev, R., Chen, G. Y. (2017). Pausing the classroom lecture: The use of clickers to facilitate student engagement. Active Learning in Higher Education, 18(2), 157-172.

Drake, J. R. (2012). A critical analysis of active learning and an alternative pedagogical framework for introductory information systems courses. Journal of Information Technology Education: Innovations in Practice, 11, 39-52.

Gleason, B. L. et al. (2011). An active-learning strategies primer for achieving abilitybased educational outcomes. Am. J. Pharm. Educ. 75(9), 186.

Linnenbrink, E.A., Pintrich, P.R. (2004). Role of affect in cognitive processing in academic contexts. In: Dai, D., Sternburg, R. (eds.)Motivation, Emotion, and Cognition, pp. 57-87. Lawrence Erlbaum Associates, Mahwah.

Massey, A. P., Brown, S. A., Johnston, J. D. (2005). It's All Fun and Games. Until Students Learn. Journal of Information Systems Education, 16 (1).

Meyers, C., Jones, T. (1993). Promoting active learning: Strategies for the college classroom. San Francisco, CA: Jossey- Bass Publishers.

Mitchell, A., Petter, S., Harris, A. (2017). Learning By Doing: Twenty Successful Active Learning Exercises for Information Systems Courses. Journal of Information Technology Eduaction: Innovations in Practice, 16(3), 21-46.

Piaget, J. (1977). The Role of Action in the Development of Thinking. In W. F. Overton, \& J. M. Gallagher, Advances in Research and Theory. New York: Plenum Press.

Ramiller, N. C. (2002). The Virtual Interactive Project: Teaching Analysis and Design Through Narrative and Drama. Communications of the Association for Information Systems, 9(1).

Thomas, D., Brown, J. S. (2011). A new culture of learning: Cultivating the imagination for a world of constant change: CreateSpace Independent Publishing.

Thongmak, M. (2017). Flipping MIS Classroom by Peers: Gateway to Student's Engagement Intention. In Proceedings of the 26th International Conference on World Wide Web Companion (pp. 387-396). International World Wide Web Conferences Steering Committee. 